\title{
Prognostic value of nuclear maspin expression for adjuvant 5-fluorouracil-based chemotherapy in advanced gastric cancer
}

\author{
KE-FENG LEI ${ }^{1,3}$, BING-YA LIU ${ }^{1}$, XIAO-LONG JIN ${ }^{2}$, YAN GUO ${ }^{2}$, MIN YE $^{2}$ and ZHENG-GANG ZHU ${ }^{1}$ \\ ${ }^{1}$ Department of Surgery, Shanghai Institute of Digestive Surgery, Ruijin Hospital, \\ Shanghai Jiao Tong University School of Medicine, Shanghai 200025; ${ }^{2}$ Department of Pathology, Ruijin Hospital, \\ Shanghai Jiao Tong University School of Medicine, Shanghai 200025; ${ }^{3}$ Department of General Surgery, \\ Zhejiang Provincial People's Hospital, Hangzhou 310014, P.R. China
}

Received November 2, 2011; Accepted March 27, 2012

DOI: $10.3892 /$ etm.2012.532

\begin{abstract}
To assess the prognostic and predictive value of maspin expression for the clinical response to 5-fluorouracil (5-FU)-based chemotherapy in advanced gastric cancer (GC) patients, the expression of maspin in primary tumors from 127 patients with advanced GC was examined using immunohistochemistry. Of the 127 patients, 74 were treated with surgery alone and 53 received additional adjuvant 5-FU-based chemotherapy. Nuclear and cytoplasmic maspin expression was observed in 46.5 (59/127) and 68.5\% (87/127) of patients, respectively. Nuclear maspin immunoreactivity was significantly associated with larger tumor size $(\mathrm{p}=0.036)$, the depth of tumor invasion $(\mathrm{p}=0.02)$ and lymph node metastasis $(p=0.002)$. Cytoplasmic maspin immunoreactivity was associated with tumor cell differentiation but not with the other clinicopathological variables. Nuclear maspin immunoreactivity had a significant association with overall survival (OS). Among the nuclear maspin-expressing patients, those who were treated with 5-FU-based adjuvant chemotherapy showed significantly longer OS than those without chemotherapy ( $\mathrm{p}=0.0004)$. In conclusion, nuclear maspin expression is associated with adverse clinical outcomes in patients with advanced GC. Patients with positive nuclear maspin expression may be more responsive to adjuvant 5-FU chemotherapy.
\end{abstract}

\section{Introduction}

Although the incidence and mortality of gastric cancer (GC) have been steadily declining over several decades in most countries, GC remains one of the most common causes of

Correspondence to: Professor Zheng-Gang Zhu, Department of Surgery, Shanghai Institute of Digestive Surgery, Ruijin Hospital, Shanghai Jiao Tong University School of Medicine, Shanghai 200025, P.R. China

E-mail: zzg1954@yeah.net

Key words: maspin, gastric cancer, prediction, immunohistochemistry, 5-fluorouracil-based chemotherapy cancer-related mortality (1). In China, 300,000 cases of mortality and 400,000 new cases associated with GC occur every year (2). Although surgical resection is essential to treat this malignancy, adjuvant (postoperative) chemotherapy should be considered for all patients who are at high risk for recurring GC and have undergone curative resection (3). Moreover, postoperative adjuvant chemotherapy that is based on fluorouracil regimens is associated with reduced risk of mortality in GC compared with surgery alone (4). Therefore, screening for GC patients who are likely to benefit from fluorouracil regimens is being investigated.

Maspin is a $42-\mathrm{kDa}$ protein that is a member of the ovalbumin clade of serine protease inhibitors (serpins). Maspin was first considered to be a tumor suppressor (5). However, conflicting opinions concerning its function in cancer progression have been reported (6-9). Maspin may have a significant role in the progression and metastasis of gastric adenocarcinoma $(10,11)$. To date, no data on the predictive value of maspin expression for fluorouracil-based chemotherapy in cases of advanced GC have been reported.

The aim of the current study was to assess the prognostic and predictive value of maspin expression for 5-fluorouracil (5-FU)-based chemotherapy in advanced GC patients.

\section{Materials and methods}

Patients and clinical features. Human GC tissues were obtained with informed consent from 127 patients with advanced GC who underwent radical resection of GC in 2000 and 2001 at the Department of Surgery at Ruijin Hospital (Shanghai, China). All diagnoses were confirmed using histopathology. The stage and grade were established using the TNM and World Health Organization classification systems. Patients who had received previous neoadjuvant chemotherapy were excluded. Among the 127 patients who underwent a radical resection, $53(41.73 \%)$ received 5-FU-based adjuvant chemotherapy (adjuvant group) and the remaining 74 (58.27\%) did not receive the treatment (surgery group) due to postoperative complications or patient preference. The chemotherapy method was performed as follows: leucovorin $(200 \mathrm{mg}$ ) was administered for two days via intravenous (i.v.) infusion prior to 5 -FU $\left(400 \mathrm{mg} / \mathrm{m}^{2}\right)$, which was administered as a $10-\mathrm{min}$ 
i.v. bolus, followed by 5 -FU $\left(600 \mathrm{mg} / \mathrm{m}^{2}\right)$ as a continuous 22 -h i.v. infusion with a light shield. This process was repeated every 3 weeks for 4 to 6 cycles. All patients were followed up systematically. The follow-up procedure included a complete history and physical examination, complete blood count, platelet count, multichannel serum chemistry analysis and additional assessments, including endoscopy and other radiological studies, every 4 months for 3 years and annually thereafter. The 127 patients included $82(64.57 \%)$ males and $45(35.43 \%)$ females, ranging from 27 to 74 years old (mean, $55.4 \pm 12.1$ years).

Immunohistochemistry (IHC). The IHC assay was performed on $4-\mu \mathrm{m}$ sections that were cut from paraffin-embedded GC samples on adhesive glass slides. The slides were deparaffinized in xylene, blocked using endogenous peroxidase in methanol with $0.3 \%$ hydrogen peroxide, washed in $0.01 \mathrm{~mol} / 1$ sodium citrate and heated in a cooker for $10 \mathrm{~min}$. Nonspecific binding sites were blocked by incubating with $10 \%$ ovalbumin The samples were incubated with primary mouse monoclonal antibody against maspin (dilution 1:50; Novocastra, Newcastle-upon-Tyne, UK) at $37^{\circ} \mathrm{C}$ for $2 \mathrm{~h}$. Negative control slides were treated without the primary antibody under equivalent conditions. For the secondary developing reagents, polymer-HRP/M/R, which was labeled using the EnVision ${ }^{\mathrm{TM}}$ System (DakoCytomation, Glostrup, Denmark), and the UltraSensitive $^{\mathrm{TM}}$ S-P (Goat) kit (Maixin Bio, Fuzhou, China) were used. The slides were developed using diaminobenzidine (DAB; DakoCytomation) and counterstained with hematoxylin.

Pathologists who were blinded to patient outcomes independently scored the immunostained slides as previously described (12). Briefly, the pathologists assigned a score for the percentage of positive-staining tumor cells $(0$, none; $1,<1 \%$; $2,1-10 \% ; 3,11-33 \% ; 4,34 \%-66 \% ; 5,>66 \%)$ and an intensity score $(0$, none; 1 , weak; 2 , intermediate; 3 , strong). These two scores were then added to obtain a IHC score for the slides. The cytoplasmic and nuclear stainings were evaluated separately. The IHC results were grouped based on the IHC score ( $<3$, negative; 4-6, weakly positive; 7-8, positive).

Statistical analysis. A $\chi^{2}$ test or two-sided Fisher's exact test was used to evaluate the statistical correlation between the maspin expression patterns and clinicopathological features of the patients. We also used univariate analysis to evaluate the correlation between the prognostic factors and overall survival (OS). Multivariate analyses were performed using the Cox regression. The differences in the mean values were evaluated using the Student's t-test. The survival curves were computed using the Kaplan-Meier method and were compared using the log-rank test. $\mathrm{P}<0.05$ was considered to indicate a statistically significant result. Statistical analyses were performed using software from SPSS 13.0 for Windows (SPSS, Inc., Chicago, IL, USA).

\section{Results}

Maspin expression and clinicopathological features. Maspin expression was detected in most patients (nuclear or cytoplasmic IHC score $\geq 4$ ). Nuclear and cytoplasmic maspin expression was detected in $46.5(59 / 127)$ and $68.5 \%$ (87/127) of patients, respectively (Fig. 1). Nuclear maspin immunoreactivity was significantly associated with larger tumor size $(\mathrm{p}=0.036)$, the depth of tumor invasion $(\mathrm{p}=0.02)$ and lymph node metastasis $(\mathrm{p}=0.002)$, but not patient age, gender or tumor cell differentiation (Table I). Cytoplasmic maspin immunoreactivity was associated with tumor cell differentiation but not with the other clinicopathological variables.

Survival prediction using clinicopathological factors. To elucidate factors that prolong survival, we performed an analysis to identify the prognostic factors for OS using the $\chi^{2}$ test or Fisher's exact test. Eight factors were analyzed, including the age and gender of the patients, tumor size, the depth of tumor invasion, lymph node metastasis, tumor cell differentiation and nuclear and cytoplasmic maspin immunoreactivity. Based on the results of these univariate analyses, five factors were significantly associated with OS (Table II): tumor size $(\mathrm{p}<0.001)$, the depth of tumor invasion $(\mathrm{p}=0.024)$, lymph node metastasis $(\mathrm{p}=0.015)$, tumor cell differentiation $(\mathrm{p}=0.022)$ and nuclear maspin immunoreactivity $(\mathrm{p}<0.001$; Table II). In multivariate analysis, nuclear maspin immunoreactivity retained an independent prognostic factor for OS $(\mathrm{p}=0.002$; Table III).

Predictive impact of nuclear maspin immunoreactivity. The predictive impact of nuclear maspin immunoreactivity was evaluated using the Kaplan-Meier survival analysis. The OS of patients with nuclear maspin expression was significantly decreased compared with that of patients without nuclear maspin expression ( $<<0.001$; Fig. 2A). Among patients with or without nuclear maspin expression, the patient survival was compared between the surgery and adjuvant groups (Fig. 2B and C). No significant difference in the OS was detected between the surgery and adjuvant groups in the 68 patients who lacked nuclear maspin expression ( $p=0.223$; Fig. 2B). In the 59 patients with nuclear maspin expression, the patients with 5-FU-based adjuvant chemotherapy had significantly longer OS than those without chemotherapy ( $<<0.001$; Fig. 2C).

\section{Discussion}

Advanced GC is incurable, but chemotherapy plays an essential role in the treatment of the disease. In the 1990s, several chemotherapy regimens were used as active agents, including 5-FU, cisplatin, methotrexate, doxorubicin, etoposide, leucovorin and mitomycin C. In the 2000s, a few new agents, including oral fluoropyrimidine (capecitabine, S-1), irinotecan, taxanes (paclitaxel, docetaxel) and oxaliplatin, were investigated for treating gastrointestinal cancers (13). Nevertheless, there is no internationally accepted regimen for the treatment of GC (14). The selection of appropriate anticancer drugs for individual patients is important. 5-FU has been widely used as a chemotherapeutic agent in GC and is considered to be one of the most effective drugs against $\mathrm{GC}$ as it mimics uracil and induces apoptosis in tumor cells (15-17). However, not all patients with advanced GC respond well to this anticancer drug. Therefore, the selection of patients with advanced GC with high chemosensitivity is crucial for the personalized therapy of GC $(18,19)$. 
Table I. Clinicopathological features with regard to maspin expression patterns.

\begin{tabular}{|c|c|c|c|c|c|c|c|c|}
\hline \multirow[b]{3}{*}{ Variable $(\mathrm{n}=127)$} & \multicolumn{8}{|c|}{ Maspin immunoreactivity } \\
\hline & \multicolumn{4}{|c|}{ Nuclear } & \multicolumn{4}{|c|}{ Cytoplasmic } \\
\hline & n (127) & Neg (68) & Pos (59) & P-value & n (127) & $\operatorname{Neg}(40)$ & Pos (87) & P-value \\
\hline \multicolumn{9}{|c|}{ Age at diagnosis (years) } \\
\hline$\leq 65$ & 92 & 48 & 44 & 0.692 & 92 & 30 & 62 & 0.831 \\
\hline$>65$ & 35 & 20 & 15 & & 35 & 10 & 25 & \\
\hline \multicolumn{9}{|l|}{ Gender } \\
\hline Male & 82 & 44 & 38 & 1 & 82 & 28 & 54 & 0.43 \\
\hline Female & 45 & 24 & 21 & & 45 & 12 & 33 & \\
\hline \multicolumn{9}{|l|}{ Tumor size $(\mathrm{cm})$} \\
\hline$\leq 5$ & 86 & 52 & 34 & 0.036 & 86 & 26 & 60 & 0.686 \\
\hline$>5$ & 41 & 16 & 25 & & 41 & 14 & 27 & \\
\hline \multicolumn{9}{|c|}{$\mathrm{T}$ (depth of invasion) } \\
\hline 2 & 40 & 28 & 12 & 0.02 & 40 & 12 & 28 & 0.96 \\
\hline 3 & 55 & 28 & 27 & & 55 & 18 & 37 & \\
\hline 4 & 32 & 12 & 20 & & 32 & 10 & 22 & \\
\hline \multicolumn{9}{|c|}{$\mathrm{N}$ (lymph node metastasis) } \\
\hline 0 & 40 & 31 & 9 & 0.002 & 40 & 10 & 30 & 0.317 \\
\hline 1 & 40 & 20 & 20 & & 40 & 11 & 29 & \\
\hline 2 & 28 & 10 & 18 & & 28 & 10 & 18 & \\
\hline 3 & 19 & 7 & 12 & & 19 & 9 & 10 & \\
\hline \multicolumn{9}{|l|}{ Differentiation } \\
\hline Differentiated & 14 & 11 & 3 & 0.052 & 14 & 8 & 6 & 0.036 \\
\hline Undifferentiated & 113 & 57 & 56 & & 113 & 32 & 81 & \\
\hline
\end{tabular}
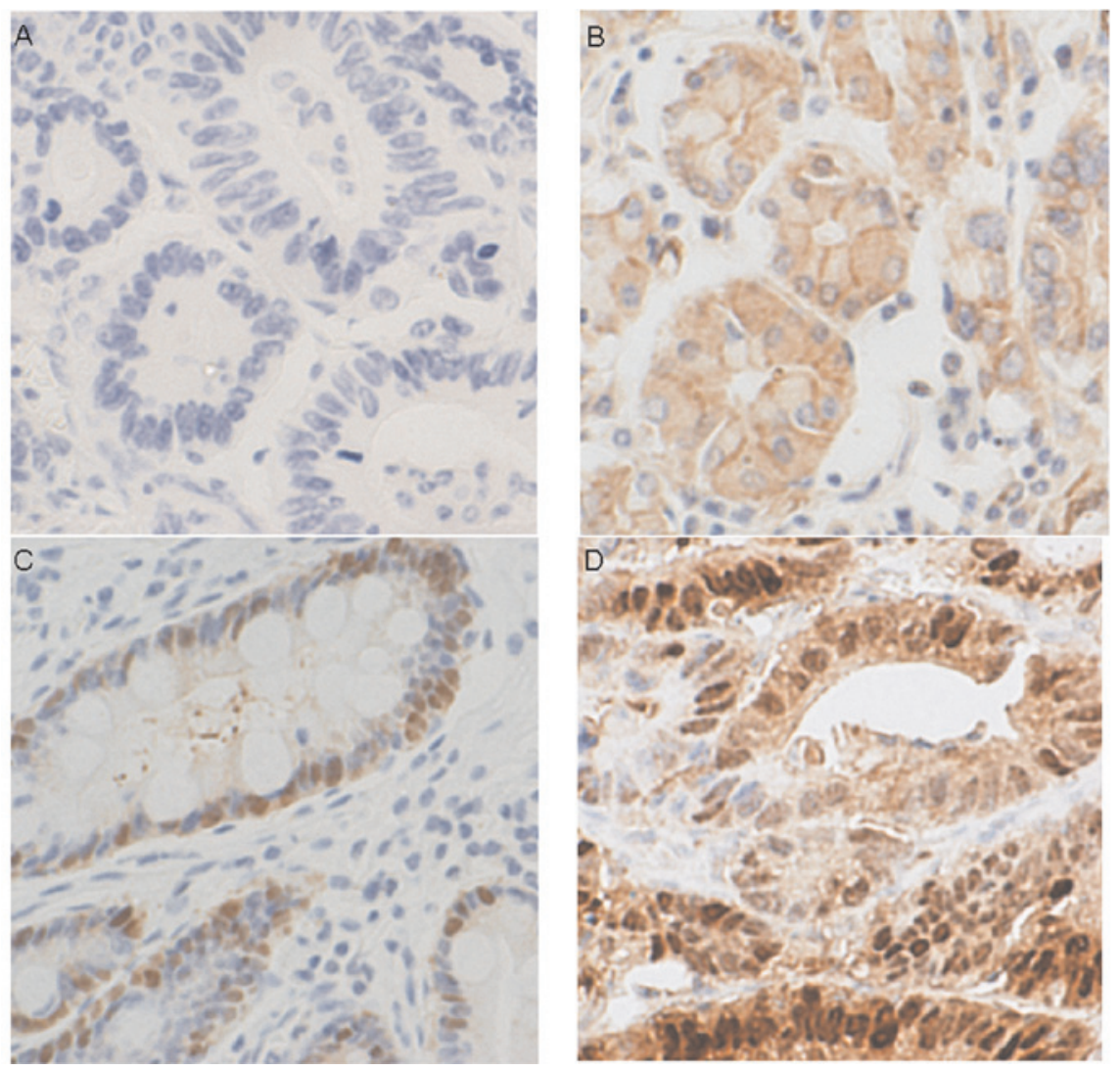

Figure 1. Representative immunohistochemical maspin staining in gastric cancer. Tumor cells with (A) negative nuclear and cytoplasmic staining, (B) negative nuclear and weakly positive cytoplasmic staining, (C) weakly positive nuclear and negative cytoplasmic staining, (D) positive nuclear and cytoplasmic staining. 
Table II. Univariate analysis of prognostic factors and overall survival.

Overall survival

Variable

n Events P-value

Age at diagnosis (years)

$\begin{array}{llll}\leq 65 & 92 & 35 & 0.122 \\ >65 & 35 & 18 & \end{array}$

Gender

Male

$82 \quad 33$

0.708

Female

$45 \quad 20$

Tumor size $(\mathrm{cm})$

$\leq 5$

$>5$

8625

4128

$\mathrm{T}$ (depth of invasion)

2

3

$40 \quad 11$

0.024

$55 \quad 23$

4

$32 \quad 19$

$\mathrm{N}$ (lymph node metastasis)

$\begin{array}{lll}0 & 40 & 11 \\ 1 & 40 & 14 \\ 2 & 28 & 16 \\ 3 & 19 & 12\end{array}$

Differentiation

Differentiated

Undifferentiated

$14 \quad 10$

0.022

11343

Nuclear maspin immunoreactivity

$\begin{array}{llll}\text { Positive } & 59 & 37 & 0 \\ \text { Negative } & 68 & 16 & \\ \text { Cytoplasmic maspin immunoreactivity } & & & \\ \text { Positive } & 87 & 34 & 0.44 \\ \text { Negative } & 40 & 19 & \end{array}$

Events, number of deaths during follow up.

Nuclear maspin expression has been reported to be associated with the response to adjuvant 5-FU-based chemotherapy in patients with stage III colon cancer (20). This finding is consis-
A

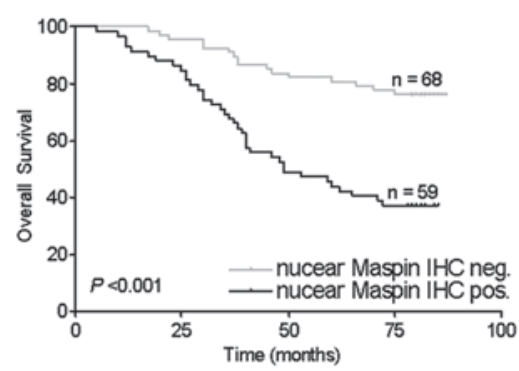

B

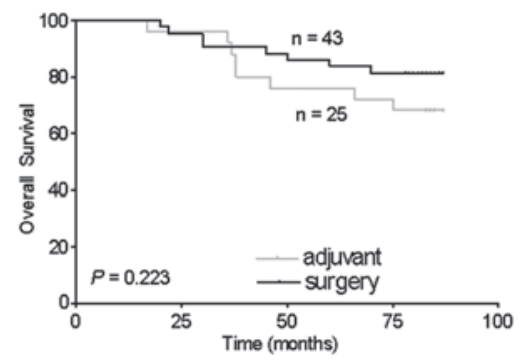

C

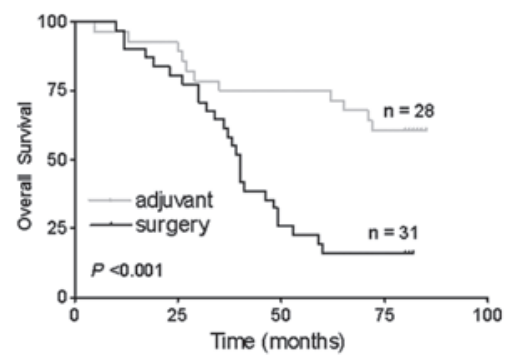

Figure 2. Distribution of time (months) to mortality as estimated using the Kaplan-Meier method. (A) Overall survival of all patients with negative and positive nuclear maspin immunoreactivity. (B and C) Subgroup analysis of overall survival for patients with (B) negative or (C) positive nuclear maspin immunoreactivity in the surgery and adjuvant therapy groups.

tent with our results showing that maspin is downregulated in fluorouracil-resistant colon cancer cells (21). However, no data on the predictive value of maspin expression in advanced GC have been reported. In the present study, samples from 127 patients with advanced GC were assessed to determine the prognostic and predictive value of maspin expression. Nuclear maspin immunoreactivity was significantly associated with clinicopathological variables, including tumor size, the depth of tumor invasion and lymph node metastasis. These clinico-

Table III. Multivariate analysis of prognostic factors and overall survival.

\begin{tabular}{lccr}
\hline Variable & Hazard ratio & 95\% confidence interval & P-value \\
\hline Age at diagnosis (years) & 1.096 & $0.604-1.986$ & 0.763 \\
Gender & 1.122 & $0.628-2.005$ & 0.697 \\
Tumor size & 4.118 & $2.014-8.419$ & 0.000 \\
T (depth of invasion) & 0.652 & $0.356-1.194$ & 0.166 \\
N (lymph node metastasis) & 1.760 & $1.197-2.589$ & 0.004 \\
Differentiation & 0.265 & $0.120-0.586$ & 0.001 \\
Nuclear maspin immunoreactivity & 2.660 & $1.438-4.919$ & 0.002 \\
Cytoplasmic maspin immunoreactivity & 0.967 & $0.527-1.774$ & 0.913 \\
\hline
\end{tabular}


pathological variables and nuclear maspin immunoreactivity had a significant association with OS. Nuclear maspin expression was an independent adverse prognostic factor for OS in advanced GC patients. However, among patients with nuclear maspin expression, the adjuvant group had significantly longer OS than the surgery group.

Maspin was first identified by subtractive hybridization and the differential display method. It was found to be expressed in normal mammary epithelial cells but not in a number of mammary carcinoma cell lines and was considered to be a tumor suppressor (5). As a tumor suppressor, maspin inhibits the motility, invasive activity and metastasis of cancer cells $(5,22-24)$ as well as angiogenesis (25). However, conflicting opinions concerning its function in cancer occurrence and progression have been reported. Maspin acts as an oncogene rather than a tumor suppressor in undifferentiated thyroid cancer, breast cancer and ductal adenocarcinoma of the pancreas (6-8). Gastric tumor specimens were found to have increased maspin expression levels compared with the corresponding normal tissues and the frequency of maspin induction was associated with the stage of GC and lymph node metastasis. Maspin may have a significant role in the progression and metastasis of gastric adenocarcinoma $(10,11)$. There are also conflicting opinions concerning the expression pattern of maspin in different types of human cancer. A previous study reported that a nuclear signal was present in $96 \%$ and a cytoplasmic signal in $35 \%$ of invasive breast cancer cases (26). Invasive ovarian cancers have been found to be more likely to have predominantly cytoplasmic staining (9). Cytoplasmic and nuclear expression of maspin has been identified in $47.6 \%$ of squamous cell cancers of the larynx (27). Two patterns of immunostaining for maspin have been observed in maspin-positive GC cases: cytoplasm-only staining $(67.0 \%)$ and staining of both cytoplasm and nucleus (33.0\%) (28); this is similar to our data, but we also found nucleus-only staining $(12.6 \%, 16 / 127$ cases) in GC cases. These different expression patterns of maspin may be due to the use of different primary antibodies, IHC protocol and tumors. It has been reported that the nuclear localization of maspin was associated with increased survival, whereas the cytoplasmic localization was associated with poor outcome in ovarian carcinoma (9), although these results were obtained without excluding the effects of adjuvant therapy. Our data indicate that nuclear maspin expression is associated with adverse clinical outcomes, but patients with positive nuclear maspin expression had a better response to adjuvant 5-FU chemotherapy. This makes nuclear maspin an attractive therapeutic target.

In the current study, we revealed that nuclear maspin expression was an independent adverse prognostic factor for patients with advanced GC. The significance of nuclear maspin expression in GC requires further study. In addition, the significance of the correlation between nuclear maspin expression and the response to adjuvant 5-FU chemotherapy remains unclear. Previous studies have revealed that the E2F1-mediated upregulation of maspin is enhanced by chemotherapeutic drugs and that the inhibition of maspin expression significantly impairs the ability of E2F1 to promote chemotherapy-induced apoptosis. Maspin mediates E2F1-induced sensitivity of cancer cells to chemotherapy (29). Whether maspin mediates E2F1-induced sensitivity of GC cells to chemotherapy or whether other factors interact with maspin in 5-FU chemotherapy requires further investigation.

In conclusion, the present study indicated that nuclear maspin expression was associated with adverse clinical outcomes in advanced GC patients. Patients with positive nuclear maspin expression may exhibit a better response to adjuvant 5-FU chemotherapy than patients with negative nuclear maspin expression. Maspin may be a new predictive marker in patients with advanced GC who are eligible for 5-FU adjuvant chemotherapy.

\section{Acknowledgements}

We would like to thank Ms. Yue-Mei Sun from the Shanghai Institute of Digestive Surgery for assistance with the patient follow-up, Mr. Jun Ji from the Shanghai Institute of Digestive Surgery for assistance with the IHC experiment and Mr. Kang Chen from the Department of Pathology, Ruijin Hospital, for assistance with the preparation of sections for the IHC experiment. This study was supported in part by the China National '863' R\&D High-Tech Key Project (2006AA02A301 and 2007AA02Z179) and the National Natural Science Foundation of China (30772107).

\section{References}

1. Jemal A, Siegel R, Ward E, et al: Cancer statistics, 2007. CA Cancer J Clin 57: 43-66, 2007.

2. Yang L: Incidence and mortality of gastric cancer in China. World J Gastroenterol 12: 17-20, 2006.

3. Macdonald JS, Smalley SR, Benedetti J, et al: Chemoradiotherapy after surgery compared with surgery alone for adenocarcinoma of the stomach or gastroesophageal junction. N Engl J Med 345: 725-730, 2001.

4. GASTRIC (Global Advanced/Adjuvant Stomach Tumor Research Collaboration) Group, Paoletti X, Oba K, Burzykowski T, et al: Benefit of adjuvant chemotherapy for resectable gastric cancer: a meta-analysis. JAMA 303: 1729-1737, 2010.

5. Zou Z, Anisowicz A, Hendrix MJ, et al: Maspin, a serpin with tumor-suppressing activity in human mammary epithelial cells. Science 263: 526-529, 1994

6. Ogasawara S, Maesawa C, Yamamoto M, et al: Disruption of cell-type-specific methylation at the Maspin gene promoter is frequently involved in undifferentiated thyroid cancers. Oncogene 23: 1117-1124, 2004.

7. Umekita Y, Ohi Y, Sagara Y, et al: Expression of maspin predicts poor prognosis in breast-cancer patients. Int J Cancer 100: 452-455, 2002.

8. Ohike N, Maass N, Mundhenke C, et al: Clinicopathological significance and molecular regulation of maspin expression in ductal adenocarcinoma of the pancreas. Cancer Lett 199: 193-200, 2003.

9. Sood AK, Fletcher MS, Gruman LM, et al: The paradoxical expression of maspin in ovarian carcinoma. Clin Cancer Res 8: 2924-2932, 2002.

10. Kim SM, Cho SJ, Jang WY, et al: Expression of maspin is associated with the intestinal type of gastric adenocarcinoma. Cancer Res Treat 37: 228-232, 2005.

11. Song SY, Son HJ, Kim MH, et al: Prognostic significance of maspin expression in human gastric adenocarcinoma. Hepatogastroenterology 54: 973-976, 2007.

12. Harvey JM, Clark GM, Osborne CK, et al: Estrogen receptor status by immunohistochemistry is superior to the ligand-binding assay for predicting response to adjuvant endocrine therapy in breast cancer. J Clin Oncol 17: 1474-1481, 1999.

13. Boku N: Perspectives for personalization in chemotherapy of advanced gastric cancer. Discov Med 9: 84-89, 2010.

14. Wagner AD, Grothe W, Haerting J, et al: Chemotherapy in advanced gastric cancer: a systematic review and meta-analysis based on aggregate data. J Clin Oncol 24: 2903-2909, 2006. 
15. Won HJ, Ha TK, Kwon SJ, et al: Differential effects of 5-fluorouracil on glucose transport and expressions of glucose transporter proteins in gastric cancer cells. Anticancer Drugs 21: 270-276, 2010.

16. Jakobsen A, Nielsen JN, Gyldenkerne N, et al: Thymidylate synthase and methylenetetrahydrofolate reductase gene polymorphism in normal tissue as predictors of fluorouracil sensitivity. J Clin Oncol 23: 1365-1369, 2005.

17. Ajani JA: Evolving chemotherapy for advanced gastric cancer. Oncologist 10 (Suppl 3): 49-58, 2005.

18. Park DJ and Lenz HJ: Determinants of chemosensitivity in gastric cancer. Curr Opin Pharmacol 6: 337-344, 2006.

19. Kubota T and Weisenthal L: Chemotherapy sensitivity and resistance testing: to be 'standard' or to be individualized, that is the question. Gastric Cancer 9: 82-87, 2006.

20. Dietmaier W, Bettstetter M, Wild PJ, et al: Nuclear Maspin expression is associated with response to adjuvant 5-fluorouracil based chemotherapy in patients with stage III colon cancer. Int J Cancer 118: 2247-2254, 2006.

21. Zheng Z,LiJ,HeX, et al:Involvement of RhoGDI2 in the resistance of colon cancer cells to 5-fluorouracil. Hepatogastroenterology 57: 1106-1112, 2010.

22. Sheng S, Truong B, Fredrickson D, et al: Tissue-type plasminogen activator is a target of the tumor suppressor gene maspin. Proc Natl Acad Sci USA 95: 499-504, 1998.
23. Dokras A, Gardner LM, Kirschmann DA, et al: The tumour suppressor gene maspin is differentially regulated in cytotrophoblasts during human placental development. Placenta 23: 274-280, 2002.

24. Shi HY, Zhang W, Liang R, et al: Modeling human breast cancer metastasis in mice: maspin as a paradigm. Histol Histopathol 18: 201-206, 2003.

25. Zhang M, Volpert O, Shi YH, et al: Maspin is an angiogenesis inhibitor. Nat Med 6: 196-199, 2000.

26. Mohsin SK, Zhang M, Clark GM, et al: Maspin expression in invasive breast cancer: association with other prognostic factors. J Pathol 199: 432-435, 2003.

27. Marioni G, Blandamura S, Giacomelli L, et al: Nuclear expression of maspin is associated with a lower recurrence rate and a longer disease-free interval after surgery for squamous cell carcinoma of the larynx. Histopathology 46: 576-582, 2005.

28. Lee DY, Park CS, Kim HS, et al: Maspin and p53 protein expression in gastric adenocarcinoma and its clinical applications. Appl Immunohistochem Mol Morphol 16: 13-18, 2008.

29. Ben Shachar B, Feldstein O, Hacohen D, et al: The tumor suppressor maspin mediates E2F1-induced sensitivity of cancer cells to chemotherapy. Mol Cancer Res 8: 363-372, 2010. 